\title{
Current and emerging treatment options for Graves' hyperthyroidism
}

This article was published in the following Dove Press journal:

Therapeutics and Clinical Risk Management

18 December 2009

Number of times this article has been viewed

\author{
Prakash Abraham' \\ Shamasunder Acharya ${ }^{2}$ \\ 'Department of Endocrinology, \\ Aberdeen Royal Infirmary, Aberdeen, \\ UK; ${ }^{2}$ Department of Endocrinology, \\ John Hunter Hospital, New Lambton \\ NSW, Australia
}

\begin{abstract}
Radioiodine, antithyroid drugs and surgery have been well established therapies for Graves' hyperthyroidism for several decades. However there remain large variations in practice among physicians in the preferred modality and the method of administration. Patient choice and perceptions also play a big role in the choice of treatment. Radioiodine may be given using fixed high doses or by calculated doses following uptake studies. The risks of radioiodine including eye disease and the role of prophylactic steroid therapy are discussed. The commonly used antithyroid drugs include carbimazole, methimazole and propylthiouracil; however a number of other agents have been tried in special situations or in combination with these drugs. The antithyroid drugs may be given in high (using additional levothyroxine in a block-replace regimen) or low doses (in a titration regimen). This review examines the current evidence and relative benefits for these options as well as looking at emerging therapies including immunomodulatory treatments such as rituximab which have come into early clinical trials. The use of antithyroid therapies in special situations is also discussed as well as clinical practice issues which may influence the choices.
\end{abstract}

Keywords: Graves' hyperthyroidism, radioiodine, antithyroid drugs, methimazole, propylthiouracil

\section{Introduction}

Graves' disease affects about $0.5 \%$ of the population, predominantly patients in the age group 40 to 60 years with a female to male ratio of 5:1 to 10:1. Circulating thyroid antibodies activate the thyroid stimulating hormone (TSH) receptor and stimulate thyroid follicular hypertrophy and hyperplasia with increases in thyroid hormone production. ${ }^{1}$ Graves' ophthalmopathy occurs in a mild form in $25 \%$ to $50 \%$ of patients with Graves' disease, with about 3\% to 5\% of patients having severe eye disease needing immunosuppression, orbital surgery or radiotherapy. ${ }^{2}$ If painless thyroiditis or toxic nodular goitre are diagnostic possibilities, a radionuclide uptake scan may be helpful to differentiate these conditions by showing increased uptake in Graves' hyperthyroidism (GH), low uptake in thyroiditis and hot nodules in toxic nodular goitre. Despite the availability of the two most popular therapeutic choices (radioiodine [RAI] and antithyroid drugs) in GH for several decades, there remain several areas of uncertainty with large differences in opinion as to the preferred modality and mode of administration. This is reflected in the large variation in the first-line choices among different countries. ${ }^{3}$ Radioiodine is used considerably more frequently in the US compared to Europe and parts of Asia. Surgery is less commonly used and is usually reserved for patients with large goitres, intolerance to antithyroid drugs and difficulties in administering RAI. 
This review will focus on the antithyroid drugs and RAI and will also touch on some of the emerging therapies.

\section{Antithyroid drugs}

Thionamides are the most commonly used antithyroid medications and were introduced in 1943 by Astwood. Methimazole (MMI), carbimazole (CBZ), and propylthiouracil (PTU) are the mainstays of antithyroid-drug therapy. MMI is the active metabolite of CBZ, and since the conversion of $\mathrm{CBZ}$ to $\mathrm{MMI}$ in the body is virtually complete, their effects and equivalent doses are thought to be comparable. These drugs block the synthesis of thyroid hormone by the thyroid gland. ${ }^{4}$ Their main effect is to inhibit thyroid hormone synthesis by interfering with thyroid peroxidase mediated iodination of tyrosine residues in thyroglobulin which is an important step in the synthesis of thyroxine (T4) and triiodothyronine (T3). ${ }^{5}$ They may also help control the disease by indirectly affecting the immune system. Various in vitro and in vivo studies have shown reductions in immune-related molecules such as intracellular adhesion molecule 1 and soluble interleukin 2 as well as decreasing antithyrotropin receptor antibodies over time. ${ }^{6-8}$ How much of these immune related improvements are due to improvements in thyroid functions is still unclear. PTU also inhibits the peripheral conversion of T4 to T3 though the effect is not clinically important in most situations.

The choice of which drug is used may be dictated by availability. CBZ is the most commonly used drug in the UK. MMI and PTU are used in the USA and MMI is used more often in most of Europe and Asia. ${ }^{5}$ The serum half-life of MMI is 6 to 8 hours whereas the half life of PTU is 1 to 2 hours. In addition MMI has been found to have measurable intrathyroidal concentrations up to 20 hours. ${ }^{9}$ Several studies have shown that MMI is effective when given in a once daily dose. ${ }^{10-12}$ PTU is not as effective in a single dose. ${ }^{13}$ The compliance with more frequent dosing schedule of PTU was only $53 \%$ compared to $83 \%$ with once daily MMI. ${ }^{11}$ These factors and the adverse effects profile of PTU have led to MMI/CBZ being the more popular first choice antithyroid drug.

\section{Dose and regimen}

The starting dose of CBZ/MMI is usually between 20 to $40 \mathrm{mg} /$ day depending on the severity of the hyperthyroidism. PTU is started at between 100 to $150 \mathrm{mg} 3$ times a day with $100 \mathrm{mg}$ of PTU considered equal to about $10 \mathrm{mg}$ of CBZ/MMI. The initial high dose of the drugs can be tapered down after 4 to 8 weeks in what is referred to as the titration regimen. A maintenance dose of 5 to $20 \mathrm{mg}$ of MMI or equivalent is achieved by about 4 to 6 months and this is continued for 12 to 18 months. The block-replace regimen refers to the option of maintaining the high dose of antithyroid drugs while adding levothyroxine to maintain euthyroidism. This has the advantage of fewer thyroid function tests (TFTs) but there is some evidence of a higher frequency of side effects. About 12 trials $^{14-25}$ (Table 1) have examined these regimens. The relapse rates are similar at over $55 \%$ but the withdrawal due to side effects (16\% versus $9 \%$ ) and complication rates

Table I Studies comparing high dose vs low dose antithyroid drugs

\begin{tabular}{|c|c|c|c|c|c|c|c|c|}
\hline \multirow[t]{2}{*}{ Study } & \multirow[t]{2}{*}{$\begin{array}{l}\text { Drug and dose } \\
\text { in high-dose arm }\end{array}$} & \multirow[t]{2}{*}{$\begin{array}{l}\text { Total no } \\
\text { assessed }\end{array}$} & \multicolumn{2}{|c|}{ Relapse (\%) } & \multicolumn{2}{|l|}{ Rash } & \multicolumn{2}{|c|}{$\begin{array}{l}\text { Withdrawal due } \\
\text { to side effects }\end{array}$} \\
\hline & & & High & Low & High & Low & High & Low \\
\hline Benker ${ }^{14}$ & MMl 40 mg & 291 & $54 \%$ & $58 \%$ & $7 \%$ & $6 \%$ & $13 \%$ & $7 \%$ \\
\hline Edmonds 15 & CBZ $60 \mathrm{mg}$ & 70 & $50 \%$ & $67 \%$ & $14 \%$ & $11 \%$ & $16 \%$ & $13 \%$ \\
\hline Goni Iriarte ${ }^{16}$ & CBZ 30 mg & 63 & $61 \%$ & $63 \%$ & & & & \\
\hline Grebe $^{17}$ & CBZ $100 \mathrm{mg}$ & 25 & $66 \%$ & $94 \%$ & $29 \%$ & $0 \%$ & $41 \%$ & $0.5 \%$ \\
\hline Jorde ${ }^{18}$ & MMI 60 mg & 41 & $58 \%$ & $77 \%$ & $21 \%$ & $7 \%$ & $31 \%$ & $15 \%$ \\
\hline Leclere ${ }^{19}$ & CBZ 60 mg & 196 & $40 \%$ & $47 \%$ & $6 \%$ & $0.8 \%$ & & \\
\hline Lucas $^{20}$ & CBZ $60 \mathrm{mg}$ & 60 & $67 \%$ & $60 \%$ & & & & \\
\hline Mclver ${ }^{21}$ & CBZ $40 \mathrm{mg}$ & 30 & $61 \%$ & $47 \%$ & & & & \\
\hline Nedrebo ${ }^{22}$ & CBZ 40 mg & 189 & $48 \%$ & $45 \%$ & $10 \%$ & $9 \%$ & & \\
\hline Rittmaster ${ }^{23}$ & MMI 30 mg & 145 & $58 \%$ & $59 \%$ & & & & \\
\hline Tuncel ${ }^{24}$ & PTU/MMI? dose & 73 & $15 \%$ & $18 \%$ & & & & \\
\hline Wilson 25 & CBZ 60 mg & 63 & $36 \%$ & $57 \%$ & $18 \%$ & $7 \%$ & & \\
\hline Total no & & 1246 & $51 \% b$ & $54 \%$ b & $9.8 \%$ & $5.8 \%$ & $16 \%$ & $9 \%$ \\
\hline
\end{tabular}

a The high dose arm added levothyroxine in a block-replace regimen while the dose was titrated down in the other arm.

'There was a high loss to follow-up of $30 \%$ in these studies and the relapse rates assuming relapsed hyperthyroidism in all dropouts were between 63 to $68 \%$.

Abbreviations: CBZ, carbimazole; MMI, methimazole; PTU, propylthiouracil. 
including rashes and agranulocytosis were higher in the block-replace regimen. ${ }^{26}$ One trial that used CBZ $100 \mathrm{mg}$ daily led to 7 out of 17 (46\%) withdrawing from the high dose arm due to side effects and 2 cases (12\%) had agranulocytosis, a potentially fatal complication. ${ }^{17}$

The addition of levothyroxine during low dose antithyroid drug therapy with continued levothyroxine alone showed promising results in one randomized trial, ${ }^{27}$ which was not confirmed in three subsequent trials ${ }^{28-30}$ or in a metaanalysis. ${ }^{26}$ The final choice of regimen is determined by the physician's preferences with the titration regimen being our preferred regimen. Whatever the choice, the reality is that close to $60 \%$ of patients will have a relapse and discussions should include the possibility of RAI or surgery as definitive options in the event of a relapse. Attempts at finding predictors for a relapse have met with variable success. Male sex, large goitres, a high FT3 level, smoking and high TSH receptor antibodies are potential relapse predictors and may also influence the choice. The majority of European patients choose antithyroid drugs for the initial therapy with a proportion choosing further antithyroid medications or RAI for subsequent relapses.

\section{Duration of therapy}

The optimal duration of therapy is considered to be 12 to 18 months. ${ }^{26}$ In one study ${ }^{31}$ the longer-duration titration regimen (18 months) had significantly fewer relapses (37\%) than the 6-month group (58\%). One study ${ }^{32}$ found there was no significant difference between 12 and 6 months using the block-replace regimen. Two other studies used longer durations of titration regimen, comparing 12 months with 24 months $^{33}$ and 18 months with 42 months of therapy. ${ }^{34}$ There was no additional benefit in these studies in continuing the antithyroid treatment beyond 18 months.

\section{Clinical practice points}

Our practice is to use a titration regimen with a starting dose of CBZ between 20 and $40 \mathrm{mg} /$ day depending on the severity of the initial thyrotoxicosis (eg, if FT4 $<30$ : CBZ 10 to $20 \mathrm{mg}$, and if FT4 > 40: CBZ: $40 \mathrm{mg}$, while also considering clinical factors of severity such as weight loss, cardiovascular involvement and duration of symptoms). We use betablockers for the initial 6 to 8 weeks if there are no contraindications. Thyroid functions are checked at 4 weeks (often by primary care saving a hospital visit) after initiation of $\mathrm{CBZ}$ and the dose of CBZ halved. Once a patient is on a maintenance dose of CBZ, TFTs are done every 2 to 4 months and the treatment continued for 12 to 18 months depending on the response. The patients are seen 4 to 6 monthly for 1 year after completion of antithyroid therapy and subsequent annual TFTs are done in primary care.

\section{Side effects of antithyroid medications}

Side effects are encountered in about $5 \%$ of patients using antithyroid medications. The most common side effect reported is drug rash, which may be noted in as high as $10 \%$ of patients using higher doses. ${ }^{26}$ Other minor side effects include arthralgia, gastrointestinal complaints, and changes in taste and smell. The side effects of MMI appear to be dose related while the side effects of PTU are less clearly related to dose. ${ }^{5}$ Minor side effects may be self limiting or may resolve with the addition of an antihistamine or switching from one antithyroid agent to the other. However the cross reactivity between the agents may be as high as 50\% and pursuing definitive therapy with RAI is the preferred option. Serious side effects that are potentially life threatening occur in about 3 of 1000 patients. Agranulocytosis (absolute granulocyte count less than 500 per $\mathrm{mL}^{3}$ ) is the most serious and occurs with a frequency of about $0.35 \%$ with the elderly being potentially at a higher risk. Most cases occur in the first 3 months; however, this can occur even a year or more after starting therapy and during renewed exposure when treating a relapse. Higher doses are associated with a greater risk and in one large series using routine monitoring of granulocyte counts, the incidence of agranulocytosis was $0.8 \%$ among 2087 patients started on MMI $30 \mathrm{mg}$ daily compared to $0.2 \%$ among 2739 patients started on MMI $15 \mathrm{mg}$ daily. ${ }^{35}$ Routine monitoring of granulocyte count is generally regarded as unhelpful by most experts. Fever and sore throat are the most common presenting features of agranulocytosis and patients should receive verbal and written information about the importance of getting an urgent white cell count in these situations and confirming the absence of this complication for continued antithyroid drug therapy. If the granulocyte count is less than $1000 / \mathrm{mL}$, the drug should be stopped. Treatment consists of the intravenous administration of antibiotics (including coverage for pseudomonas which is regarded as the most frequent infection) in patients who are febrile or have obvious infections. The administration of granulocyte colony-stimulating factor is considered to shorten the recovery time and length of hospitalization, though one prospective randomized trial failed to confirm this. ${ }^{36}$ There is cross reactivity between PTU and MMI for agranulocytosis and further use of these agents is contraindicated.

Hepatotoxicity is rarer than agranulocytosis with a frequency of $0.1 \%$ to $0.2 \% .^{5}$ Asymptomatic elevation of 
aminotransferases occurs in untreated hyperthyroid patients and during commencement of PTU and usually normalizes. Routine monitoring of liver functions is not recommended. PTU-related hepatotoxicity usually occurs after 3 months of therapy and takes the form of an allergic hepatitis with marked elevation of liver enzymes. A fatality rate of $25 \%$ to $50 \%$ has been reported though this may well be due to a reporting bias. Appropriate management of severe hepatic failure with specialist involvement is required. The hepatic toxicity of MMI is typical of a cholestatic process and generally recovers slowly following discontinuation of the therapy.

Vasculitis is another major side effect seen more commonly with PTU. Some patients especially Asians develop antineutrophil cytoplasmic antibody (ANCA) positive vasculitis with resultant renal failure, arthritis, vasculitic rash and respiratory symptoms. Steroids, cyclophosphamide and hemodialysis may be needed in some patients, while others improve on drug discontinuation. A cross sectional study in the UK showed that $33 \%$ of patients on PTU, $16 \%$ on CBZ and $30 \%$ of previously antithyroid drug-treated Graves' patients had ANCA positivity compared to about 5\% of euthyroid controls and untreated Graves' patients. ${ }^{37}$ The clinical significance of these findings is not known.

\section{Special situations}

\section{Pregnancy and lactation}

Hyperthyroidism occurs in $0.1 \%$ to $0.2 \%$ of all pregnancies. Untreated hyperthyroidism results in increased risk of pre-eclampsia, preterm delivery, low birth weight and increased neonatal mortality and morbidity. The mother is also at increased risk of heart failure, thyroid storm and pre-eclampsia. PTU is the preferred antithyroid agent during pregnancy, as congenital anomalies such as aplasia cutis (single or multiple lesions of 0.5 to $3 \mathrm{~cm}$ at the vertex or occipital area in the scalp), choanal and esophageal atresia are reported more frequently with MMI. ${ }^{38}$ The incidence of these anomalies is quite rare and it is acceptable to continue MMI particularly in areas where PTU is not easily available. The PTU dose is reduced to the lowest effective dose to maintain the FT4 towards the upper end of the reference range with monthly monitoring of thyroid functions. ${ }^{39}$ The activity levels of Graves' disease may fluctuate during pregnancy, with exacerbation during the first trimester with improvement in later pregnancy with a higher chance of an exacerbation soon after delivery. Up to a third of patients may be able to discontinue the PTU during the later part of pregnancy. Thyroid functions need to be checked at 6 and 12 weeks postpartum in anticipation of potential postpartum exacerbation of the GH. In patients who have adverse effects with antithyroid drugs or require persistently high doses, thyroid surgery may be considered during the second trimester. Thyroid receptor antibodies should be measured by the end of the second trimester in patients who have current Graves' disease or have had previous ablation (RAI or surgery). Patients with elevated antibody levels need assessment for foetal and neonatal thyroid dysfunction. Foetal ultrasound features of thyroid dysfunction include growth restriction, advanced bone age, goitre or cardiac failure. Both PTU and MMI appear in low concentrations in the breast milk but do not influence the infant thyroid function and breast feeding is permitted on moderate doses of these agents.

\section{Adolescence}

It is not uncommon to continue the antithyroid medications beyond the usual 12 to 18 months during the critical years of the child's education and examinations. An opportune timing is chosen mutually with the child and parents to withdraw the medication such that a recurrence in the first few months following withdrawal is least likely to cause a major disruption to studies and progress.

\section{Elderly}

Longer-term low dose CBZ/MMI is acceptable in some elderly patients when other modalities of definite therapy such as RAI or surgery are less appropriate. The care needs of elderly patients may prevent the use of RAI due to the difficulty in administering this while minimizing contact with vulnerable carers. The risks of cardiac failure and atrial fibrillation with uncontrolled hyperthyroidism as well as the challenges of regular monitoring in this age group are additional considerations. No significant complications were noted in one study giving continuous low dose MMI for over 10 years. ${ }^{40}$

\section{Inability to take oral preparations}

In patients unable to take antithyroid medications orally, rectal and parenteral routes of administration have been reported though there are no commercially available formulations. One report used an enema formulation of PTU made of eight $50 \mathrm{mg}$ tablets dissolved in mineral oil. ${ }^{41}$ Another report used an enema preparation of PTU with sterile water administrated by means of a Foley catheter inserted into the rectum. ${ }^{42} \mathrm{MMI}$ has also been administered rectally. ${ }^{43}$ One study compared the use of an enema preparation with water versus a suppository preparation with polyethylene glycol base and found comparable therapeutic effects. ${ }^{44}$ 


\section{Atrial fibrillation}

Atrial fibrillation due to hyperthyroidism is associated with increased risk of thromboembolism. ${ }^{45}$ Therefore in addition to rate control agents such as betablockers, anticoagulation with warfarin is highly recommended. Meticulous control of hyperthyroidism is best achieved initially with antithyroid drugs. Once hyperthyroidism is well controlled and rhythm stabilized, definitive therapy with RAI should be considered to eliminate hyperthyroidism permanently. Anitocoagulation may be affected by thyroid hormone status and methimazole, and therefore careful monitoring is essential. Anticoagulation should be continued till hyperthyroidism is permanently eliminated and sinus rhythm is restored.

\section{Radioiodine for $\mathrm{GH}$}

Radioiodine (RAI) emerged as a major treatment for hyperthyroidism after Hertz and Roberts successfully treated hyperthyroid patients with ${ }^{131} \mathrm{I}$ in the US in $1941 .{ }^{46}$ Despite vast worldwide clinical experience for 6 decades, controversies exist in almost every area of its use. Expert opinion is still divided on the timing of RAI, ${ }^{47}$ dosing of RAI, its long-term effects and the use of adjunctive antithyroid drugs.

\section{Mechanism of action}

After oral administration, RAI is absorbed and is incorporated in to thyroid. Continuous radiation to thyroid cells results in extensive local destruction and thyroid gland is ablated over a period of 6 to 18 weeks. ${ }^{48}$

\section{RAI dosing}

\section{Low dose versus high dose RAI}

Low dose RAI is aimed at curing hyperthyroidism without resulting in hypothyroidism. However, this approach results in higher treatment failures ${ }^{49}$ needing additional antithyroid drug treatment and further RAI doses. ${ }^{49,50}$ Although the incidence of hypothyroidism was low immediately after RAI with this approach, more than $50 \%$ of patients still developed hypothyroidism in the long term. On the contrary, high dose (4.7 to $5.7 \mathrm{MBq} / \mathrm{g}$ thyroid tissue) results in higher cure rate but most patients would become hypothyroid. ${ }^{51}$ As such, 20\% of patients with GH develop hypothyroidism due to natural history of the disease. ${ }^{52}$ The incidence of hypothyroidism in patients who achieve euthyroid state following RAI is around 3\% per annum. Hypothyroidism is preferable to hyperthyroidism as it is easier to treat and hyperthyroidism is associated with serious morbidities such as atrial fibrillation and osteoporosis. Therefore, when treating patients with GH with RAI, it is desirable to cure hyperthyroidism even if the dose required to achieve this induces iatrogenic hypothyroidism.

\section{Fixed versus calculated dose of RAI}

In the fixed dose regimens, RAI is administered without taking thyroid volume or RAI uptake into consideration. Doses ranging from $185 \mathrm{MBq}$ to $550 \mathrm{MBq}$ of RAI are commonly administered, with higher doses resulting in higher cure rates. In the second approach, a 24-hour uptake of RAI is measured before treatment and the dose is calculated based on the RAI uptake. Although this approach is individualized and ensures optimal dosing and prevents under treatment, it requires an additional trip to specialist centers and local expertise to measure the uptake. In one randomized controlled trial $(\mathrm{RCT})^{53}$ the success rate as defined by elimination of hyperthyroidism was higher (71\%) in fixed dose with $555 \mathrm{MBq}$ compared with $58 \%$ in the calculated dose (to deliver $100 \mathrm{~Gy}$ ) arm. However, success rate was inversely related to thyroid size in the fixed dose regime. For smaller thyroid glands with volumes $15 \mathrm{~mL}$ or less, success rate was $100 \%$ whereas success rate was only $25 \%$ when thyroid volume was more than $75 \mathrm{~mL}$. In the calculated dose regime, success rate was $45 \%$ with thyroid volumes $75 \mathrm{~mL}$ or more. In clinical practice, $\mathrm{GH}$ is usually associated with smaller thyroid goiters compared with multinodular goiters, and therefore a fixed dose regime works well for most patients. Our practice is to use a fixed dose treatment with $550 \mathrm{MBq}$. A higher dose (up to $800 \mathrm{MBq}$ ) may be required for patients with very large goiters with Graves'.

\section{Adjunctive antithyroid drugs with RAI}

RAI therapy may result in worsening of thyrotoxic symptoms due to an increase in thyroid hormone concentrations in the first few weeks after therapy. This may result in adverse cardiac outcomes such as atrial fibrillation. Thyroid storm is rare but can occur after RAI. Pre-treatment with an antithyroid drug restores euthyroidism and may limit such adverse outcomes, especially in older patients and in those with severe hyperthyroidism. Although this approach will not prevent a rise in thyroid hormone levels following RAI, it may be argued that the rise of thyroid hormone occurs from a lower baseline. ${ }^{54}$ Similarly, some centers use post-treatment antithyroid drugs with RAI. Antithyroid medications are started a few days following RAI and are continued for 4 to 6 weeks. However it has been suggested that adjunctive antithyroid drugs reduce the efficacy of RAI. The evidence for this was reviewed in a recently published meta-analysis involving 1306 participants in 14 RCTs. ${ }^{55}$ 
Antithyroid drugs given in the week before and after RAI increased the risk of treatment failure by $28 \%$ but lowered the risk of hypothyroidism. There were 158/442 events of treatment failure in the antithyroid drugs arm compared with 116/435 in the control arm. In these RCTs CBZ (in 3 RCTS), MMI (in 6) and PTU (in 4) were used, while 1 RCT with 2 treatment arms used PTU and MMI. The dose of RAI used varied from 158 and $585 \mathrm{MBq}$. Further sensitivity analyses showed higher risk of treatment failure with fixed dose regimens compared with calculated dose methods, pre treatment with antithyroid drugs and higher doses of antithyroid drugs. The incidence of atrial fibrillation was $1 / 660(0.2 \%)$ in the adjunctive antithyroid group versus $3 / 646(0.5 \%)$ in the control group with RAI alone. Mortality was reported to be higher in the RAI-alone group (6/646, 0.9\%) compared with $1 / 660(0.2 \%)$ in the adjunctive antithyroid group. No further details were available on increased mortality seen in the RAI-alone group. Previous studies have shown increased risk of treatment failure with propylthiouracil ${ }^{56}$ but this was not proven in the meta-analyses. The mechanism by which antithyroid drugs increase the risk of treatment failure is not clear but is thought to be due to decreased iodine uptake in the pretreatment group and inhibition of thyroid peroxidase involved in cell death after $\mathrm{RAI}^{57}$ in the post-treatment group.

Some studies have evaluated the use of lithium as an adjunct to RAI to facilitate iodine uptake. In a controlled study, lithium carbonate $885 \mathrm{mg}$ daily for 2 weeks with RAI increased radiation dose by $40 \%{ }^{58}$ The role of lithium as an adjunct to RAI was evaluated in an RCT involving 110 newly diagnosed patients with GH. Lithium $900 \mathrm{mg} /$ day started on the day of RAI for 6 days increased hyperthyroidism cure rate ( $72 \% \mathrm{RAI} ; 83 \%$ lithium with $\mathrm{RAI})$ and controlled hyperthyroidism earlier with better goiter shrinkage. ${ }^{59}$ There were no significant side effects from short-term lithium use in this study. However large-scale RCTs are required before lithium can be used routinely. At present lithium can be considered in patients with larger goiters with Graves', when thyroidectomy is not possible or for those patients where RAI failed previously. Glucocorticoids have also been evaluated for their role as an adjunct to RAI but have not been shown to be effective in improving hyperthyroidism cure rates. ${ }^{60}$

\section{Clinical practice points}

Our practice is to render patients euthyroid before RAI with antithyroid drugs and we discontinue antithyroid drugs a week before planned RAI. We administer $550 \mathrm{MBq}$ RAI as a fixed dose and routinely do not restart antithyroid drugs following RAI. We use nonselective betablockers in high risk patients for about 6 to 8 weeks. If using antithyroid drugs after RAI, a minimal effective dose of CBZ/MMI should be used starting 3 days after RAI for 4 for 6 weeks with periodic monitoring of thyroid hormone levels. Antithyroid drugs should be discontinued as soon as free thyroid hormones fall below the normal reference range or TSH start rising. Transient hypothyroidism after RAI is common, especially when lower doses of RAI are used. Long-term thyroxine therapy is recommended for permanent hypothyroidism and this is more likely when higher doses of RAI are used.

\section{Use of recombinant TSH in GH}

Recombinant TSH (rhTSH) has been found to increase RAI uptake when used for ablating thyroid remnant for patients with differentiated thyroid cancer following surgery. Theoretically it is possible to use rhTSH in patients with $\mathrm{GH}$ to increase RAI uptake and ablate thyroid successfully. The use of rhTSH has been evaluated in patients with multinodular goiter $^{61}$ with success. However, it is expensive and may cause acute worsening of hyperthyroidism. RAI as such has more than $80 \%$ success rate in Graves' patients and therefore rhTSH is not recommended.

\section{Short- and long-term effects of RAI Short term}

RAI is usually well tolerated but occasionally patients experience discomfort in the neck. Rarely patients experience enlarging goiter, which may lead to airway obstruction. High dose steroid therapy usually resolves the inflammatory response associated with radiation thyroiditis. A small minority of patients develop clinically significant worsening of hyperthyroidism including thyroid storm.

Elevated thyroid hormones may result in atrial fibrillation and adverse cardiac outcomes. Steroid-responsive Hashimoto encephalopathy (diffuse encephalopathy and raised thyroid antibodies) following RAI has been reported in the literature. ${ }^{62}$ Occasionally RAI can induce $\mathrm{GH}$ in patients with multinodular goiter.

\section{RAl and the risk of ophthalmopathy}

One of the main concerns in using RAI is the risk of developing or worsening of ophthalmopathy. We published a systematic review of 10 RCTs involving 1136 patients comparing the effects of RAI on ophthalmopathy with antithyroid drugs and surgery. ${ }^{63}$ Compared with antithyroid drugs, RAI increased risk of ophthalmopathy by 4 -fold. The use of RAI 
also increased the risk of severe ophthalmopathy (defined as ophthalmopathy requiring immunosuppression, orbital radio therapy, surgery). For patients with mild pre-existing ophthalmopathy, prednisolone prophylaxis $(0.5 \mathrm{mg} / \mathrm{kg}$ body weight starting with RAI, continued for 6 weeks after) was highly effective in preventing worsening of ophthalmopathy. In fact, in some patients ophthalmopathy improved after RAI. Adjunctive antithyroid drugs with RAI made no difference to the ophthalmopathy outcome. Smoking, high levels of pre-treatment T3 (more than twice the upper limit of normal) and post-RAI hypothyroidism were associated with increased risk of ophthalmopathy on subgroup analysis.

\section{Clinical practice points}

The risk of developing ophthalmopathy is around $20 \%$ after RAI and around 5\% after antithyroid drugs; the risk of severe ophthalmopathy is around 7\% after RAI. We recommend appropriate counseling before consenting and strong advice to give up smoking. Thyroid functions should be monitored regularly after RAI and any hypothyroidism should be treated promptly to reduce risk of ophthalmopathy. At least 12 months follow-up after RAI is essential as ophthalmopathy usually develops or worsens around 6 months after RAI but can occur up to 2 years. Use prednisolone 20 to $40 \mathrm{mg}$ daily for 6 weeks starting a day before RAI in patients with mild ophthalmopathy and perhaps in smokers and those with high T3 levels.

\section{Long-term effects of RAI}

Hypothyroidism is the main long-term effect of RAI. Several studies have reported a small but significantly increased risk of cardiovascular- ${ }^{64,65}$ and cancer-related mortality ${ }^{65,66}$ in patients treated with RAI, although it is unknown whether this is due to the effect of hyperthyroidism or due to therapy with RAI. In the West Midlands study, 67 excess deaths during 15,968 person-years follow-up after RAI (standardized mortality ratio 1.14 ) were mainly due to cardiovascular causes. ${ }^{64}$ Interestingly, this excess mortality was not seen in patients who were on thyroxine replacement therapy following RAI. Untreated hypothyroidsm following RAI was associated with increased risk of ischemic heart disease and death. In a more recent Finnish study, ${ }^{65}$ all-cause mortality was found to be 453 vs 406 per 10,000 person-years in patients treated with RAI and controls, respectively, (rate ratio 1.12). Cerebrovascular disease accounted for most deaths but death due to cancer was also increased. Gastrointestinal tract cancers, particularly cancer of the stomach and esophagus, were increased suggesting a possible local effect of RAI after oral administration. Mortality increased with increased dose of RAI.
However, overall mortality was increased only in patients with nodular goiter and not in Graves' patients.

On the contrary, other studies ${ }^{67,68}$ found no increase in overall cancer incidence and mortality but noted a small relative increase in thyroid cancer, although the absolute risks remained low. The increased incidence of thyroid cancer following RAI is mainly in patients with multinodular goiter, suggesting risk is largely due to nodular disease itself rather than RAI. Athough the long-term follow-up data are reassuring in children and adolescents with GH treated with RAI ${ }^{69}$ we recommend cautious use of RAI in very young patients with $\mathrm{GH}$.

\section{Contraindications to RAI}

Pregnancy (or immediate desire) and lactation are the major contraindications for RAI. Pregnancy must be excluded before RAI and women should be strictly advised to defer pregnancy for at least 6 months after RAI. ${ }^{70}$ This is essential not only to avoid fetal damage by radiation but also to ensure hyperthyroidism is cured and hypothyroidism is promptly corrected well before pregnancy to avoid adverse fetal outcome. Inadvertent RAI treatment before 12 weeks of gestation is usually not associated with fetal thyroid dysfunction but exposure after 12 weeks can ablate fetal thyroid. Early pregnancy radiation exposure may result in miscarriage or fetal malformations. However, the absolute risks of such complications are uncertain and termination of pregnancy is not routinely recommended. ${ }^{39}$

Although evidence is limited, men should be advised to defer fathering for at least 4 months after RAI due to radiation potentially affecting spermatogenesis. ${ }^{39}$ Severe ophthalmopathy is generally considered to be a contraindication for RAI.

\section{RAI in special circumstances}

Additional precautions will be required for patients with end stage renal disease who are undergoing hemodialysis and those living in institutions such as prisons. RAI therapy has been successfully used in children to treat GH from as early as 2 years of age. ${ }^{71}$

\section{Radiation protection advice for patient undergoing RAI}

Radiation protection guidelines differ across different countries, which may influence the choice of therapy. Patients who are undergoing RAI should be appropriately counseled regarding radiation protection safety. They should be advised to avoid close contacts with young children and pregnant 
women for 2 weeks after RAI. They should also take precautions to keep their clothes and utensils separately for the first few days after RAI. They must report to their treating centers immediately if they start vomiting or become incontinent of urine within 24 hours after RAI, for safe disposal of excreta. Patients should be advised to report to airport authorities of their treatment as security alarms could be triggered off with radiation. ${ }^{72}$ It is useful to issue a treatment card to patients confirming the date of RAI, the dose and the duration for which radiation protections apply.

\section{Other antithyroid agents Betablockers}

Many of the symptoms of hyperthyroidism such as sweating, anxiety, tremor and palpitations are caused by increased sympathetic activity and can be controlled rapidly by betablockers. Propranolol in relatively high doses of over $160 \mathrm{mg}$ per day can mildly inhibit conversion of T4 to T3. Once daily betablockers such as atenolol 50 to $100 \mathrm{mg}$ or nadolol 40 to $80 \mathrm{mg}$ can be used to improve compliance. In the absence of contraindications such as asthma, betablockers are used in the first few weeks of treating hyperthyroidism while awaiting the effect of antithyroid medications. They may also be used when antithyroid medications are withdrawn for treating with RAI. Rate-limiting calcium channel blockers may be used if there are contraindications for betablockers.

\section{lodine and iodine-containing compounds}

These are rarely used for the rapid control of hyperthyroidism in the context of thyroid storm or in the preoperative preparation for thyroid surgery. Iodide decreases thyroid hormone synthesis by blocking iodide oxidation and organification - the Wolff-Chaikoff effect. It also inhibits thyroglobulin proteolysis and release of T4 and T3. The effect is rapid and pronounced but lasts for only a few weeks with a potential for subsequent deterioration. Iodide decreases the vascularity of the thyroid but in one controlled study had no significant influence on blood loss or perioperative course. ${ }^{73}$ It can be given as Lugol's solution ( $8 \mathrm{mg}$ of saturated iodide per drop) 3 to 5 drops 3 times a day or as a saturated solution of potassium iodide (SSKI, $50 \mathrm{ng}$ of iodide per drop) 1 drop 3 times a day. Oral cholecystographic agents (sodium iopanoate and sodium ipodate) have also been used for rapidly lowering thyroid hormone levels in combination with $\mathrm{MMI}^{74}$ and may be useful in thyroid storm.

\section{Lithium}

Lithium has a role in inhibiting thyroid hormone synthesis and release. Lithium can rarely be used in patients intolerant of thionamides. It has been shown to reduce the thyroid hormone increase after thionamide withdrawal and RAI therapy in Graves' disease as discussed above.

\section{Potassium perchlorate}

This is a competitive inhibitor of iodide transport but is rarely used due to its side effects, particularly the risk of aplastic anemia with long-term use. It may be used in the context of amiodarone-induced thyrotoxicosis or while awaiting RAI in patients allergic to thionamides.

\section{Cholestyramine}

Cholestyramine decreases the enterohepatic reabsorbtion of thyroid hormones. Thyrotoxic patients have an abnormal increase of thyroid hormones in their enterohepatic circulation. Cholestyramine used in combination with PTU or MMI brought about a more rapid decline of thyroid hormones during the first month of antithyroid therapy. ${ }^{75,76}$

\section{Newer immunomodulatory agents}

Graves' disease is an autoimmune B-cell mediated condition in which TSH receptor antibodies (TRAb) play an important role in the pathogenesis. A few agents are being investigated but the only agent that has entered phase II clinical studies is rituximab (RTX). This is an anti-CD20 monoclonal antibody which causes B cell depletion in the circulation as well as in target organs such as the thyroid. RTX was the first monoclonal antibody used in the treatment of non-Hodgkin's lymphoma. This has also been used in various autoimmune diseases including rheumatoid arthritis. Two small studies have shown a positive benefit in the clinical course in Graves' ophthalmopathy without affecting the thyroid function. ${ }^{77,78}$ The effects on the hyperthyroidism in Graves' disease are less promising. In one controlled study 4 of 10 patients receiving RTX remained in remission 1 year after therapy while all 10 patients not receiving RTX relapsed..$^{79}$ In a further phase II study of 13 patients with relapsing Graves' disease, 9 patients given 2 doses of RTX $1000 \mathrm{mg}$ intravenously with a 2-week interval were euthyroid at a median of 18 months of follow-up while 4 patients who had initial high thyroid hormone levels failed to respond and needed subsequent RAI therapy. ${ }^{80}$ Due to the limited experience and costs of these agents, further studies are needed before these agents are considered as possible therapies in $\mathrm{GH}$.

\section{Surgery and interventional therapies for $\mathrm{GH}$}

Thyroid surgery is an option that may be used particularly as an alternative to RAI during recurrent hyperthyroidism 


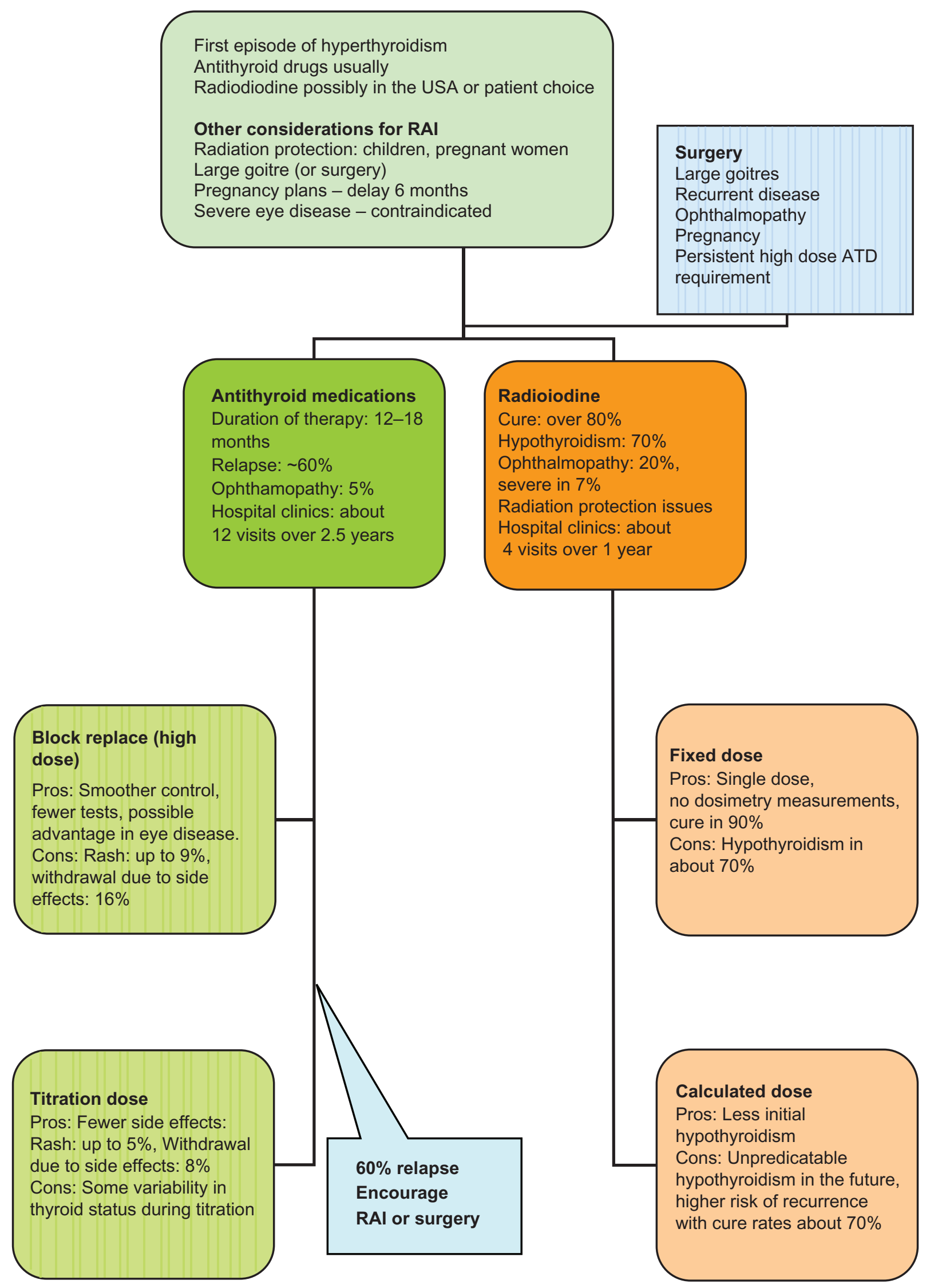

Figure I Evidence-informed pragmatic choices of antithyroid therapy. Abbreviations: ATD, antithyroid drugs; RAI, radioiodine. 
or in uncontrolled hyperthyroidism despite high doses of antithyroid drugs. This may also be considered during poorly controlled hyperthyroidism during pregnancy or if there is a suspicion of a coexistent malignant thyroid nodule. Patients with large goiters particularly with compressive symptoms, with poor compliance with antithyroid drugs, or with severe ophthalmopathy would also be suitable candidates for a surgical approach. ${ }^{81}$ There has been some debate about the extent of surgery - total versus subtotal thyroidectomy. There has been 1 meta-analysis ${ }^{82}$ on these approaches. In 35 studies involving over 7000 patients, total thyroidectomy was equally safe, with $0.9 \%$ recurrent laryngeal nerve palsy (RLN), 0.9\% permanent hypoparathyroidism and no recurrent disease. Subtotal thyroidectomy resulted in $0.7 \%$ RLN palsy, $1.0 \%$ incidence of hypoparathyroidism and $7.9 \%$ recurrent or persistent disease. If surgery was being considered for the therapy of Graves' disease, total thyroidectomy would be our preferred option. This would also be preferred in the presence of mild Graves' ophthalmopathy. Surgery may also induce a rise in thyroid receptor antibodies and thyroid hormones, and steroid cover may need to be considered for those with significant ophthalmopathy. One randomized study suggested that in patients with ophthalmopathy on glucocorticoids, total thyroid ablation (surgery followed by RAI) had a better outcome in relation to the progression of the ophthalmopathy than total thyroidectomy alone. ${ }^{83}$ Selective arteriography, followed by embolization of the thyroid arteries was a novel technique reported in 1 series, 14 of 22 patients with Graves' disease becoming euthyroid with no additional therapy. ${ }^{84}$

\section{Treatment of complications of $\mathrm{GH}$}

\section{Graves' ophthalmopathy}

The main aim of treatment of Graves' ophthalmopathy is to restore euthyroidism promptly since both hyper and hypothyroidism aggravates ophthalmopathy. Patients should be strongly advised to give up smoking. In addition, local measures such as lubricants help to relieve symptoms. Prisms may help diplopia. Moderate to severe cases should be referred to ophthalmologists for specialist management. A detailed discussion of the treatment of Graves' ophthalmopathy is beyond the scope of this article.

\section{Graves' dermopathy}

Treatment of dermopathy associated with Graves' disease is unsatisfactory especially in its chronic phase. Topical steroids with occlusive dressing or systemic glucocorticoids may help recent onset of dermopathy.

\section{Choice of therapy (Figure I)}

The three main choices in the form of surgery, RAI and antithyroid drugs have remained unchanged over several decades. Various factors influence the choice of treatment, which include patient and physician preference, availability of surgical expertise, concerns on radiation or the difficulties in complying with radiation protection guidance, concerns on side effects of medications, predictors of relapsing disease and cost. RAI is the most cost-effective treatment when considering the cost of surgery and the high relapse rates. ${ }^{85}$ However in the European context most patients would prefer to have at least one course of antithyroid medications before considering RAI. Our approach would be to discuss all three possibilities at the outset. Most patients would choose antithyroid drugs and we would use the titration regimen for 12 to 18 months. We would attempt to be more persuasive about RAI at the point of the second relapse. Women may also be influenced by the fact that radiation protection may be technically challenging once they have had children, and if their hyperthyroidism is tending to have relapses they may well wish to consider a definitive route if they have no immediate plans for pregnancy. If RAI is chosen we would be using a fixed dose and consider steroid cover only if there was evidence of mild ophthalmopathy. We would be encouraging modifying risk factors such as smoking at every opportunity. Following RAI we would aim to pick up the hypothyroidism and treat this without delay. In the proportion who refuse RAI we would give another 12- to 18-month course of antithyroid drugs and in some with the risk of recurrent hyperthyroidism (cardiac failure, atrial fibrillation or elderly) we would consider a much longer course of low dose CBZ. The patient would also have the opportunity to choose surgery particularly if they have a large goitre or have significant thyroid eye disease.

\section{Disclosures}

The authors declare no conflicts of interest.

\section{References}

1. Brent GA. Graves' Disease. N Engl J Med. 2008;358:2594-2605.

2. Wiersinga WM, Bartalena L. Epidemiology and prevention of Graves' ophthalmopathy. Thyroid. 2002;12:855-860.

3. Vaidya B, Williams GR, Abraham P, Pearce SHS. Radioiodine treatment for benign thyroid disorders: results of a nationwide survey of UK endocrinologists. Clin Endocrinol (Oxf). 2008;68:814-820.

4. Nayak B, Hodak SP. Hyperthyroidism. Endocrinol Metab Clin N Am. 2007;36:617-656.

5. Cooper D. Antithyroid drugs. N Engl J Med. 2005;352:905-917.

6. Sonnet E, Massart C, Gibassier J, et al. Longitudinal study of soluble intracellular adhesion molecule-1 (ICAM-1) in sera of patients with Graves' disease. J Endocrinol Invest. 1999;22:430-435.

7. Tsatsoulis A, Vlachoyiannopoulos PG, Dalekos GN, et al. Increased serum interleukin-1 beta during treatment of hyperthyroidism with antithyroid drugs. Eur J Clin Invest. 1995;25:654-658. 
8. McGregor AM, Peterson MM, Mclachlan SM, et al. Carbimazole and the autoimmune response in Graves' disease. N Engl J Med. 1980;303: 302-307.

9. Jansson R, Dahlberg PA, Johansson H, Lindström B. Intrathyroidal concentrations of methimazole in patients with Graves' disease. J Clin Endocrinol Metab. 1983;57:129-132.

10. Shiroozu A, Okamura K, Ikenoue H, et al. Treatment of hyperthyroidism with a single daily dose of methimazole. J Clin Endocrinol Metab. 1986; 63:125-128.

11. Nicholas WC, Fischer RG, Stevenson RA, et al. Single daily dose of methimazole compared to every 8 hours propylthiouracil in the treatment of hyperthyroidism. South Med J. 1995;88:973-976.

12. Bouma DJ, Kammer H. Single daily dose methimazole treatment of hyperthyroidism. West J Med. 1980;132:13-15.

13. He CT, Hsieh AT, Kuo SW, et al. Comparison of single daily dose methimazole and propylthiouracil in the treatment of Graves' hyperthyroidism. Clin Endocrinol (Oxf). 2004;60:676-681.

14. Benker G, Reinwein D, Kahaly G, et al. Is there a methimazole dose effect on remission rate in Graves' disease? Results from a long-term prospective study. Clin Endocrinol (Oxf). 1998;49:451-457.

15. Edmonds CJ, Tellez M. Treatment of Graves' disease by carbimazole: high dose with thyroxine compared to titration dose. Eur J Endocrinol. 1994;131:120-124.

16. Goni-Iriarte MJ, Forga Llenas L, Iriarte Berioiz A, et al. Recurrence of Graves-Basedow disease: the influence of treatment schedule. [Spanish]. Medicina Clinica 1995;104:11-14.

17. Grebe SK, Feek CM, Ford HC, et al. A randomized trial of short-term treatment of Graves' disease with high-dose carbimazole plus thyroxine versus low-dose carbimazole. Clin Endocrinol (Oxf). 1998;48: 585-592.

18. Jorde R, Ytre-Arne K, Stromer J, Sundsfjord J. Short-term treatment of Graves' disease with methimazole in high versus low doses. J Intern Med. 1995;238:161-165.

19. Leclere J. Treatment of Basedow disease with synthetic antithyroid drugs. Evaluation of the dose on the efficacy of the long term treatment. [French]. Annales de Endocrinologie 1994;55:11-14.

20. Lucas A, Salinas I, Rius F, et al. Medical therapy of Graves' disease: does thyroxine prevent recurrence of hyperthyroidism? J Clin Endocrinol Metab. 1997;82:2410-2413.

21. McIver B, Rae P, Beckett G, Wilkinson E, Gold A, Toft A. Lack of effect of thyroxine in patients with Graves' hyperthyroidism who are treated with an antithyroid drug. N Engl J Med. 1996;334:220-224.

22. Nedrebo BG, Holm P, Uhlving S, et al. Graves' disease: drug regimens and predictors of outcome. Eur J Endocrinol. 2002;147: 583-589.

23. Rittmaster RS, Abbott EC, Douglas R, et al. Effect of methimazole, with or without L-thyroxine, on remission rates in Graves' disease. J Clin Endocrinol Metab. 1998;83:814-818.

24. Tuncel E, Ertturk E, Imamoglu S. Effects of Antithyroid drugs alone and plus L-Thyroxine on the relapse of Graves disease. [abstract] J Endocrinol Invest. 2000;7:23.

25. Wilson R, Buchanan L, Fraser WD, McKillop JH, Thomson JA. Do higher doses of carbimazole improve remission in Graves' disease? QJ Med. 1996;89:381-385.

26. Abraham P, Avenell A, Watson WA, Park CM, Bevan JS. A systematic review of drug therapy for Graves' hyperthyroidism. Eur J Endocrinol. 2005;153:489-498.

27. Hashizume K, Ichikawa K, Sakurai A, et al. Administration of thyroxine in treated Graves' disease. Effects on the level of antibodies to thyroid-stimulating hormone receptors and on the risk of recurrence of hyperthyroidism. N Engl J Med. 1991;324: 947-953.

28. Glinoer D, de Nayer P, Bex M; Belgian Collaborative Study Group on Graves' Disease. Effects of 1-thyroxine administration, TSH-receptor antibodies and smoking on the risk of recurrence in Graves' hyperthyroidism treated with antithyroid drugs: a double-blind prospective randomized study. Eur J Endocrinol. 2001;144:475-483.
29. Hoermann R, Quadbeck B, Roggenbuck U, et al; for Basedow Study Group. Relapse of Graves' disease after successful outcome of antithyroid drug therapy: results of a prospective randomized study on the use of levothyroxine. Thyroid. 2002;12:1119-1128.

30. Mastorakos G, Doufas AG, Mantzos E, Mantzos J, Koutras DA. T4 but not $\mathrm{T} 3$ administration is associated with increased recurrence of Graves' disease after successful medical therapy. J Endocrinol Invest. 2003;26:979-984.

31. Allannic H, Fauchet R, Orgiazzi J, et al. Antithyroid drugs and Graves' disease: a prospective randomized evaluation of the efficacy of treatment duration. J Clin Endocrinol Metab. 1990;70:675-679.

32. Weetman AP, Pickerill AP, Watsob P, Chatterjee VK, Edwards OM. Treatment of Graves' disease with the block-replace regimen of antithyroid drugs: the effect of treatment duration and immunogenetic susceptibility on relapse. QJ Med. 1994;87:337-341.

33. García-Mayor RV, Páramo C, Luna Cano R, Pérez Mendez LF, Galofré JC, Andrade A. Antithyroid drug and Graves' hyperthyroidism. Significance of treatment duration and TRAb determination on lasting remission. J Endocrinol Invest. 1992;15:815-820.

34. Maugendre D, Gatel A, Campion L, et al. Antithyroid drugs and Graves' disease - prospective randomized assessment of long-term treatment. Clin Endocrinol (Oxf). 1999;50:127-132.

35. Takata K, Kubota S, Fukata S, et al. Methimazole-induced agranulocytosis in patients with Graves' disease is more frequent with an initial dose of $30 \mathrm{mg}$ daily than with $15 \mathrm{mg}$ daily. Thyroid. 2009;19: 559-563.

36. Fukata S, Kuma K, Sugawara M. Granulocyte colony-stimulating factor (G-CSF) does not improve recovery from antithyroid drug-induced agranulocytosis: a prospective study. Thyroid. 1999;9(1):29-31.

37. Harper L, Chin L, Daykin J, et al. Propylthiouracil and carbimazoleassociated antineutrophil cytoplasmatic antibodies (ANCA) in patients with Graves' disease. Clin Endocrinol (Oxf). 2004;60:671-675.

38. Mandel S, Cooper DS. The use of antithyroid drugs in pregnancy and lactation. J Clin Endocrinol Metab. 2001;86:2354-2359.

39. Abalovich M, Amino N, Barbour LA, et al. Management of thyroid dysfunction during pregnancy and postpartum: an Endocrine Society Clinical Practice Guideline. J Clin Endocrinol Metab. 2007;92(Suppl 2): S1-S47.

40. Azizi F, Ataie L, Hedayati M, Mehrabi Y, Shiekholeslami F. Effect of long-term continuous methimazole treatment of thyrotoxicosis: comparison with radioiodine. Eur J Endocrinol 2005;152:695-701.

41. Walter RM, Bartle WR. Rectal administration of propylthiouracil in the treatment of Graves' disease. Am J Med. 1990;88:69-70.

42. Yeung S, Go R, Balasubramanyam A, et al. Rectal administration of iodide and propylthiouracil in the treatment of thyroid storm. Thyroid. 1995;5:403-405.

43. Nabil N, Miner DJ, Amatruda JM. Methimazole: an alternative route of administration. J Clin Endocrinol Metab. 1982;54:180-181.

44. Jongjaroenprasert W, Akarawut W, Chantasart D, Chailurkit L, Rajatanavin R. Rectal administration of propylthiouracil in hyperthyroid patients: comparison of suspension enema and suppository form. Thyroid. 2002;12:627-631.

45. Osman F, Gammage MD, Franklyn JA. Hyperthyroidism and cardiovascular morbidity and mortality. Thyroid. 2002;12:483-487.

46. Sawin CT, Becker DV. Radioiodine and the treatment of hyperthyroidism: the early history. Thyroid. 1997;7:163-176.

47. Wartofsky L, Glinoer D, Solomon B, et al. Differences and similarities in the diagnosis and treatment of Graves' disease in Europe, Japan and the United States. Thyroid. 1991;1:129-135.

48. Franklyn JA. The management of hyperthyroidism. $N$ Engl J Med. 1994;330:1731-1738.

49. Sridama V, McCormick M, Kaplan EL, Fauchet R, DeGroot LJ. Longterm follow-up study of compensated low-dose ${ }^{131} \mathrm{I}$ therapy for Graves' disease. N Engl J Med. 1984;311:426-432.

50. Goolden AW, Stewart JS. Long-term results from graded low dose radioactive iodine therapy for thyrotoxicosis. Clin Endocrinol (Oxf). $1986 ; 24: 217-222$. 
51. Alexander EK, Larsen PR. High dose of (131)I therapy for the treatment of hyperthyroidism caused by Graves' disease. J Clin Endocrinol Metab. 2002;87:1073-1077.

52. Tamai H, Kasagi K, Takaichi Y, et al. Development of spontaneous hypothyroidism in patients with Graves' disease treated with antithyroidal drugs: clinical, immunological, and histological findings in 26 patients. J Clin Endocrinol Metab. 1989;69:49-53.

53. Peters H, Fischer C, Bogner U, Reiners C, Schleusener H. Radioiodine therapy of Graves' hyperthyroidism: standard vs calculated ${ }^{131}$ iodine activity. Results from a prospective, randomized, multicentre study. Eur J Clin Invest. 1995;25:186-193.

54. Burch HB, Solomon BL, Cooper DS, Ferguson P, Walpert N, Howard R. The effect of antithyroid drug pretreatment on acute changes in thyroid hormone levels after ${ }^{131}$ I ablation for Graves' disease. J Clin Endocrinol Metab. 2001;86(7):3016-3021.

55. Walter MA, Briel M, Christ-Crain M, et al. Effects of antithyroid drugs on radioiodine treatment: systematic review and meta-analysis of randomised controlled trials. BMJ. 2007;10;334:514.

56. Imseis RE, Vanmiddlesworth L, Massie JD, Bush AJ, Vanmiddlesworth NR. Pretreatment with propylthiouracil but not methimazole reduces the therapeutic efficacy of iodine- ${ }^{-131}$ in hyperthyroidism. J Clin Endocrinol Metab. 1998;83:685-687.

57. Wilson R, McKillop JH, Buchanan LM, et al. The effect of carbimazole therapy on interleukin 2, interleukin 2 receptor and free radicals. Autoimmunity. 1990;8:3-7.

58. Dunkelmann S, Kunstner H, Nabavi E, Eberlein U, Groth P, Schumichen C. Lithium as an adjunct to radioiodine therapy in Graves' disease for prolonging the intrathyroidal effective half-life of radioiodine. Useful or not? Nuclear-Medizin. 2006;45:213-218; quiz N51-N52.

59. Bogazzi F, Bartalena L, Brogioni S, et al. Comparison of Radioiodine with Radioiodine plus Lithium in the treatment of Graves' hyperthyroidism. J Clin Endocrinol Metab. 1999:84;2:499-503.

60. Jensen BE, Bonnema SJ, Hegedüs L. Glucocorticoids do not influence the effect of radioiodine therapy in Graves' disease. Eur J Endocrinol. 2005;153:15-21.

61. Silva MN, Rubió IG, Romão R, et al. Administration of a single dose of recombinant human thyrotrophin enhances the efficacy of radioiodine treatment of large compressive multinodular goitres. Clin Endocrinol (Oxf). 2004;60:300-308.

62. Dihné M, Schuier FJ, Schuier M, et al. Hashimoto encephalopathy following iodine $131\left({ }^{131} \mathrm{I}\right)$ radiotherapy of Graves disease. Arch Neurol. 2008; $65: 282-283$.

63. Acharya SH, Avenell A, Philip S, Burr J, Bevan JS, Abraham P. Radioiodine therapy (RAI) for Graves' disease (GD) and the effect on ophthalmopathy: a systematic review. Clin Endocrinol (Oxf). 2008;69: 943-950.

64. Franklyn JA, Sheppard MC, Maisonneuve P. Thyroid function and mortality in patients treated for hyperthyroidism. JAMA. 2005;294:71-80.

65. Metso S, Jaatinen P, Huhtala H, Auvinen A, Oksala H, Salmi J. Increased cardiovascular and cancer mortality after radioiodine treatment for hyperthyroidism. J Clin Endocrinol Metab. 2001;92:2190-2196.

66. Hall P, Berg G, Bjelkengren G, et al. Cancer mortality after iodine-131 therapy for hyperthyroidism. Int J Cancer. 1992;50:886-890.

67. Franklyn JA, Maisonneuve P, Sheppard M, Betteridge J, Boyle P. Cancer incidence and mortality after radioiodine treatment for hyperthyroidism: a population-based cohort study. Lancet. 1999;353:2111-2115.
68. Ron E, Doody MM, Becker DV, et al. Cancer mortality following treatment for adult hyperthyroidism. Cooperative Thyrotoxicosis Therapy Follow-up Study Group. JAMA. 1998;280:347-355.

69. Read Jr CH, Tansey MJ, Menda Y. A 36-year retrospective analysis of the efficacy and safety of radioactive iodine in treating young Graves' patients. J Clin Endocrinol Metab. 2004;89:4229-4233.

70. Royal College of Physicians, UK. Radioiodine in the management of benign thyroid disease. Clinical guidelines. URL: http://www.rcplondon. ac.uk/pubs/brochure. aspx?e=208.

71. Ma C, Kuang A, Xie J, Liu G. Radioiodine treatment for pediatric Graves' disease. Cochrane Database Syst Rev. 2008;(3):CD006294.

72. Sinzinger H, Aiginger P, Neumann I, Havlik E. Radiation alarm at an airport after radioiodine therapy. Nucl Med Commun. 2005;26:67-68.

73. Coyle PJ, Mitchell JE. Thyroidectomy: is Lugol's iodine necessary? Ann R Coll Surg Engl. 1982;64:344-345.

74. Roti E, Robuschi G, Gardini E, et al. Comparison of methimazole, Methimazole and sodium ipodate, and methimazole and saturated solution of potassium iodide in the early treatment of hyperthyroid Graves' disease. Clin Endocrinol (Oxf). 1988;28:305-314.

75. Mercado M, Mendoza-Zubieta V, Bautista-Osorio R, et al. Treatment of hyperthyroidism with a combination of methimazole and cholestyramine. J Clin Endocrinol Metab. 1996;81:3191-3193.

76. Tsai WC, Pei D, Wang T, et al. The effect of combination therapy with propylthiouracil and cholestyramine in the treatment of Graves' hyperthyroidism. Clin Endocrinol (Oxf). 2005;62:521-524.

77. Salvi M. Vannucchi G. Campi I, et al. Treatment of Graves' disease and associated opthalmopathy with the anti-CD20 monoclonal antibody rituximab: An open study. Eur J Endocrinol. 2007;156:33-40.

78. El Fassi D, Nielsen CH, Hasselbalch HC, et al. Treatment resistant severe active Graves' ophthalmopathy successfully treated with B lymphocyte depletion. Thyroid. 2006;16:709-710.

79. El Fassi D, Nielsen CH, Bonnema SJ, Hasselbalch HC, Hegedus L. B lymphocyte depletion with the monoclonal antibody rituximab in Graves' disease: A controlled pilot study. J Clin Endocrinol Metab. 2007;92:1769-1772.

80. Heemstra KA, Toes RE, Sepers J, et al. Rituximab in relapsing Graves' disease, a phase II study. Eur J Endocrinol. 2008;159:609-615.

81. Stalberg P, Svensson A, Hessman O, Akerstrom G, Hellman P. Surgical Treatment of Graves' Disease: Evidence-Based Approach. World J Surg. 2008;32:1269-1277.

82. Palit TK, Miller CC III, Miltenburg DM. The efficacy of thyroidectomy for Graves' disease: a meta-analysis. J Surg Res 2000;90:161-165.

83. Menconi F, Marino M, Pinchera A, et al. Effects of total thyroid ablation versus near-total thyroidectomy alone on mild to moderate Graves' orbitopathy treated with intravenous glucocorticoids. J Clin Endocrinol Metab. 2007;92:1653-1658.

84. Xiao H, Zhuang W Wang S, et al. Arterial embolization: A nover approach to thyroid ablative therapy for Graves' disease. $J$ Clin Endocrinol Metab. 2002;87:3583-3589.

85. Patel NN, Abraham P, Buscombe J, Vanderpump MPJ. The Cost Effectiveness of Treatment Modalities for Thyrotoxicosis in a UK Centre. Thyroid. 2006;16:593-598.
Therapeutics and Clinical Risk Management

\section{Publish your work in this journal}

Therapeutics and Clinical Risk Management is an international, peerreviewed journal of clinical therapeutics and risk management, focusing on concise rapid reporting of clinical studies in all therapeutic areas, outcomes, safety, and programs for the effective, safe, and sustained use of medicines. This journal is indexed on PubMed Central, CAS,

\section{Dovepress}

EMBase, Scopus and the Elsevier Bibliographic databases. The manuscript management system is completely online and includes a very quick and fair peer-review system, which is all easy to use. Visit $\mathrm{http}: / /$ www.dovepress.com/testimonials.php to read real quotes from published authors. 